\title{
On symmetry group classification of a fin equation
}

\author{
Özlem Orhan', Gülden Gün ${ }^{1}$ and Teoman Özer ${ }^{2 *}$
}

*Correspondence: tozer@itu.edu.tr ${ }^{2}$ Division of Mechanics, Faculty of Civil Engineering, İstanbul Technical University, Maslak, İstanbul, 34469, Turkey

Full list of author information is available at the end of the article

\begin{abstract}
We deal with the Noether symmetry classification of a nonlinear fin equation, in which thermal conductivity and heat transfer coefficient are assumed to be functions of the temperature. In this study Noether symmetries of the fin equation are investigated using the partial Lagrangian approach. This classification includes Noether symmetries, first integrals and some invariant solutions with respect to different choices of thermal conductivity and heat transfer coefficient functions.
\end{abstract}

Keywords: Noether symmetries; partial Lagrangian approach; classification; first integrals; nonlinear fin equation

\section{Introduction}

The aim of the present study is to classify the Noether point symmetries of a fin equation. In the literature, symmetry classifications of differential equations with respect to Lie point symmetries and Noether symmetries have an important role for understanding possible solutions of differential equations [1-11]. Noether symmetries can also be used in finding the first integrals (conserved forms) of the nonlinear problems. The earliest studies on Noether symmetries based on the Noether theorem are due to German mathematician Emily Noether [1]. Applications of the Noether theorem to differential equations can provide some important information about the problems in mechanics, physics, and engineering sciences [12-15]. In order to apply the Noether theorem, the differential equations should have a standard Lagrangian. On the other hand, one can apply the partial Lagrangian method to differential equations to investigate Noether symmetries and first integrals by using Euler-Lagrange equations [12]. Here, we determine the partial Lagrangian and Noether symmetries of the fin equation by applying partial Noether approach to a nonlinear fin equation.

This study is organized as follows. In Section 2, we present some fundamental definitions of the Euler-Lagrange operator, partial Lagrangian and partial Noether operators. In Section 3 we discuss the nonlinear fin equation and the corresponding determining equations. This section also includes different cases corresponding to different choices of thermal conductivity and heat transfer coefficient. Furthermore, Noether point symmetries and first integrals for each different case are presented. Section 4 presents some invariant solutions, and the last section summarizes some important results in the study. 


\section{Preliminaries}

Suppose that $x$ is the independent variable and $y=\left(y^{1}, \ldots, y^{m}\right)$ is the independent variable with coordinates $y^{\alpha}$ with respect to $x$ given in the following form:

$$
y_{x}^{\alpha}=y_{1}^{\alpha}=D_{x}\left(y^{\alpha}\right), \quad y_{s}^{\alpha}=D_{x}^{s}\left(y^{\alpha}\right), \quad s \geq 2, \alpha=1,2, \ldots, m,
$$

where $D_{x}$ is the total derivative operator [2-7], with respect to $x$, which is defined as

$$
D_{x}=\frac{\partial}{\partial x}+y_{x}^{\alpha} \frac{\partial}{\partial y^{\alpha}}+y_{x x}^{\alpha} \frac{\partial}{\partial y_{x}^{\alpha}} \text {. }
$$

Here, the vector space of all differential functions of all finite orders is represented by $\mathcal{A}$ that is a universal space. Also, operators apart from the total derivative operator (2.2) are defined on the space $\mathcal{A}$.

Definition 1 The operator

$$
\frac{\delta}{\delta y^{\alpha}}=\frac{\partial}{\partial y^{\alpha}}+\sum_{s \geq 1}\left(-D_{x}\right)^{s} \frac{\partial}{\partial y_{x}^{\alpha}}, \quad \alpha=1,2, \ldots, m
$$

is called the Euler operator or Euler-Lagrange operator.

Definition 2 The generalized operator is given by

$$
X=\xi \frac{\partial}{\partial x}+\eta^{\alpha} \frac{\partial}{\partial y^{\alpha}}+\sum_{s \geq 1} \xi_{s}^{\alpha} \frac{\partial}{\partial y_{s}^{\alpha}}
$$

where

$$
\xi_{s}^{\alpha}=D_{x}^{s}\left(W^{\alpha}\right)+\xi y_{s+1}^{\alpha}, \quad s \geq 2, \alpha=1,2, \ldots, m,
$$

and $W^{\alpha}$ is the Lie characteristic function

$$
W^{\alpha}=\eta^{\alpha}-\xi y_{x}^{\alpha}, \quad \alpha=1,2, \ldots, m
$$

Here we can rewrite the generalized operator (2.4) in terms of a characteristic function as follows:

$$
X=\xi D_{x}+W^{\alpha} \frac{\partial}{\partial y^{\alpha}}+\sum_{s \geq 1} D_{x}^{s}\left(W^{\alpha}\right) \frac{\partial}{\partial y_{s}^{\alpha}},
$$

and the Noether operator associated with a generalized operator $X$ can be defined

$$
N=\xi+W^{\alpha} \frac{\partial}{\partial y^{\alpha}}+\sum_{s \geq 1} D_{x}^{s}\left(W^{\alpha}\right) \frac{\partial}{\partial y_{s}^{\alpha}} .
$$

Now let us consider a $k$ th-order system of an ordinary differential equation

$$
E_{\alpha}\left(x, u, u_{(1)}, u_{(2)}, \ldots, u_{(k)}\right)=0, \quad \alpha=1,2, \ldots, m
$$


Definition 3 The first integral of the system $I \in \mathcal{A}(2.8)$ can be written in the form

$$
D_{x} I=0 .
$$

Then the expression (2.9) is called the local conservation law for system (2.8). Furthermore, $D_{x} I=\mathrm{Q}^{\alpha} E_{\alpha}$ is called the characteristic form of conservation law (2.9) where the functions $\mathrm{Q}^{\alpha}=\left(\mathrm{Q}^{1}, \ldots, \mathrm{Q}^{m}\right)$ are the associated characteristics of the conservation law (2.9).

Definition 4 [12] Let $L=L\left(x, u, u_{(1)}, u_{(2)}, \ldots, u_{(\alpha)}\right) \in \mathcal{A}, \alpha \leq k$, let nonzero functions $f_{\alpha}^{\beta} \in \mathcal{A}$ be a partial Lagrangian, and let $X$ be a Lie-Bäcklund operator of the form of (2.4). If there exists a vector $B \in \mathcal{A}, B \neq N L+C, C=$ constant, we have the following relation:

$$
X_{(\alpha)} L+L D_{x}(\xi)=W^{\alpha} \frac{\delta L}{\delta y^{\alpha}}+D_{x}(B)
$$

where $W=\left(W^{1}, \ldots, W^{m}\right), B(x, y)$ is the gauge function, and $W^{\alpha} \in \mathcal{A}$, then $X$ is called a partial Noether operator corresponding to $L$, and $X_{(\alpha)}$ is the $\alpha$ th prolongation of the generalized operator (2.7). If we apply the Euler-Lagrange operator (2.3) to the Lagrangian $L$, then we obtain the following differential equations:

$$
\frac{\delta L}{\delta u^{\alpha}}=0, \quad \alpha=1,2, \ldots, m,
$$

which are called Euler-Lagrange equations and the Lagrangian $L$ is called a standard Lagrangian. However, if $\frac{\delta L}{\delta u^{\alpha}} \neq 0$, the Lagrangian $L$ is called as a partial Lagrangian and the corresponding differential equations are called partial Euler-Lagrange equations.

Definition $5 X$ is a Noether point symmetry corresponding to a Lagrangian of the system of differential equations (2.8) if there exists a function $B(x, y)$. In addition, $X$ is a Noether point symmetry corresponding to a Lagrangian of the fin equation, then $I$ is the first integral associated with $X$, which is given by the expression [12]

$$
I=\xi L+\left(\eta-y^{\prime} \xi\right) L_{y^{\prime}}-B
$$

\section{Noether symmetries of a fin equation}

We now consider the Noether symmetry classification of the nonlinear fin equation [15-17]

$$
y^{\prime \prime}+\frac{K^{\prime}(y)}{K(y)}\left(y^{\prime}\right)^{2}-\frac{H(y)}{K(y)}=0
$$

where $K$ and $H$ are thermal conductivity and heat transfer coefficient, respectively, which are considered as functions of temperature, and $y=y(x)$ is the temperature function and $x$ is a dimensional spatial variable. The Lie point symmetries equation (3.1) is investigated in the reference [18]. In this study, we consider the partial Noether approach to analyze Noether symmetries of equation (3.1). 
For fin equation (3.1), we can write the Euler-Lagrange operator (2.3)

$$
\frac{\delta}{\alpha y^{\alpha}}=\frac{\partial}{\partial y^{\alpha}}-D_{x} \frac{\partial}{\partial y_{x}}+D_{x}^{2} \frac{\partial}{\partial y_{x x}}
$$

and the partial Lagrangian $L$ for fin equation (3.1) can be written as

$$
L=\frac{1}{2}\left(y^{\prime}\right)^{2}+\int \frac{H(y)}{K(y)} d y
$$

and if we apply Euler-Lagrange operator (3.2) to Lagrangian (3.3), then we obtain

$$
\frac{\delta L}{\delta y}=\frac{H(y)}{K(y)}-y^{\prime \prime}
$$

In addition, if we rewrite the fin equation in the form

$$
-y^{\prime \prime}+\frac{H(y)}{K(y)}=\left(y^{\prime}\right)^{2} \frac{K^{\prime}(y)}{K(y)}
$$

then equation (3.4) becomes

$$
\frac{\delta L}{\delta y}=\left(y^{\prime}\right)^{2} \frac{K^{\prime}(y)}{K(y)} .
$$

In relation (2.10), the partial Lagrangian (3.3) has at most first order derivatives, and then we can take $\alpha=1$ and write the following definition:

$$
W^{1} \frac{\delta L}{\delta y}=\left(\eta-\xi y^{\prime}\right)\left(y^{\prime 2} \frac{K^{\prime}(y)}{K(y)}\right)=\eta y^{\prime 2} \frac{K^{\prime}(y)}{K(y)}-\xi y^{\prime 3} \frac{K^{\prime}(y)}{K(y)},
$$

and $D_{x}(B)$ is defined in the form

$$
D_{x}(B)=B_{x}+y^{\prime} B_{y} .
$$

By application of the first prolongation of the generalized operator (2.7) $X_{(1)}$ to Lagrangian (3.3), we get

$$
X_{(1)} L=\eta \frac{H(y)}{K(y)}+\eta^{1} y^{\prime},
$$

where $\eta^{1}$ is defined in the form [2-7]

$$
\eta^{1}=\eta_{x}+\left(\eta_{y}-\xi_{x}\right) y^{\prime}-\xi_{y}\left(y^{\prime}\right)^{2} .
$$

The expansion of the form of (2.10) by using the definition of the first prolongation of the Noether operator and relations (3.6)-(3.10) is written as follows:

$$
\begin{aligned}
& \eta_{x} y^{\prime}+\left(\eta_{y}-\xi_{x}\right) y^{\prime 2}-\xi_{y} y^{\prime 3}+\frac{1}{2} \xi_{x} y^{2}+\frac{1}{2} \xi_{y} y^{\prime 3}+\xi_{x} \int \frac{H(y)}{K(y)} d y \\
& \quad+\xi_{y} y^{\prime} \int \frac{H(y)}{K(y)} d y+\eta \frac{H(y)}{K(y)}+\xi \frac{K^{\prime}(y)}{K(y)} y^{\prime 3}-\eta \frac{K^{\prime}(y)}{K(y)} y^{\prime 2}-B_{x}-y^{\prime} B_{y}=0 .
\end{aligned}
$$


The usual separation by powers of derivatives of $y$ (3.11) reduces to the following determining equations:

$$
\begin{aligned}
& -\frac{1}{2} \xi_{y}+\xi \frac{K^{\prime}(y)}{K(y)}=0, \\
& \eta_{y}-\frac{1}{2} \xi_{x}-\eta \frac{K^{\prime}(y)}{K(y)}=0, \\
& \eta_{x}+\xi_{y} \int \frac{H(y)}{K(y)} d y-B_{y}=0, \\
& \xi_{x} \int \frac{H(y)}{K(y)} d y+\eta \frac{H(y)}{K(y)}-B_{x}=0 .
\end{aligned}
$$

To find the infinitesimals $\xi$ and $\eta$, the determining equations (3.12)-(3.15) should be solved together. First, from the solution of equation (3.12), we have that

$$
\xi=K(y)^{2} a(x)
$$

where $a(x)$ is a function of $x$. The solution of equation (3.13) is

$$
\eta=\frac{1}{2} a^{\prime}(x) K(y) \int K(y) d y+K(y) b(x)
$$

where $b(x)$ is a function of $x$. Thus, if we differentiate (3.14) with respect to $x$ and (3.15) with respect to $y$, then we can eliminate the function $B(x, y)$ from equations (3.14)-(3.15), and we obtain the following single equation:

$$
\begin{gathered}
\left(\frac{1}{2} a^{\prime}(x) \int K(y) d y+b(x)\right) H^{\prime}(y)+\frac{3}{2} K(y) a^{\prime}(x) H(y) \\
-\frac{1}{2} a^{\prime \prime \prime}(x) K(y)\left(\int K(y) d y\right)-K(y) b^{\prime \prime}(x)=0,
\end{gathered}
$$

which is a differential equation including unknown functions $K(y), H(y), a(x)$ and $b(x)$. Using equations (3.16)-(3.18), one can classify Noether symmetries and the corresponding first integrals of nonlinear fin equation (3.1) based on different forms of the thermal conductivity $K(y)$ and the heat transfer coefficient $H(y)$ and differential relations for $a(x)$ and $b(x)$.

Case 1: $K(y)=k($ constant $)$.

In equation (3.19), if we consider $K(y)=k$ (constant), then we obtain the following differential equation for $H(y)$ function:

$$
\left(2 b(x)+a^{\prime}(x) k y\right) H^{\prime}(y)+3 k a^{\prime}(x) H(y)-k\left(2 b^{\prime \prime}(x)-k a^{\prime \prime \prime}(x)\right)=0 .
$$

Now we analyze differential equation (3.19) for different $H(y)$ functions corresponding to different solutions of (3.18), and we get differential relations between functions $a(x)$ and $b(x)$, which give Noether symmetries and the corresponding first integral for each case. 
Case 1.1: $H(y)=h($ constant $)$.

For this case, equation (3.19) becomes

$$
3 h k a^{\prime}(x)-2 k b^{\prime \prime}(x)-k^{2} y a^{\prime \prime \prime}(x)=0 .
$$

In (3.20) it is clear that $a^{\prime \prime \prime}(x)=0,3 h a^{\prime}(x)-2 b^{\prime \prime}(x)=0$. From the solutions of $a(x)$ and $b(x)$, we obtain the following infinitesimal functions:

$$
\begin{aligned}
& \xi=k^{2}\left(c_{1}+x c_{2}+x^{2} c_{3}\right), \\
& \eta=\frac{1}{2} k^{2} y\left(c_{2}+2 x c_{3}\right)+k\left(\frac{3}{4} h x^{2} c_{2}+\frac{1}{2} h x^{3} c_{3}+c_{4}+x c_{5}\right),
\end{aligned}
$$

and the corresponding Noether symmetries

$$
\begin{aligned}
& X_{1}=k^{2} \frac{\partial}{\partial x}, \quad X_{2}=k^{2} x \frac{\partial}{\partial x}+\left(\frac{1}{2} k^{2} y+\frac{3}{4} k h x^{2}\right) \frac{\partial}{\partial y}, \\
& X_{3}=k^{2} x^{2} \frac{\partial}{\partial x}+\left(k^{2} x y+\frac{1}{2} h k x^{3}\right) \frac{\partial}{\partial y}, \quad X_{4}=k \frac{\partial}{\partial y}, \quad X_{5}=k x \frac{\partial}{\partial y} .
\end{aligned}
$$

By using relations (3.14) and (3.15), the function $B(x, y)$ is found in the form

$$
\begin{aligned}
B(x, y)= & \frac{1}{4} h^{2} x^{3} c_{2}+\frac{3}{2} h k x y c_{2}+\frac{1}{8} h^{2} x^{4} c_{3} \\
& +\frac{3}{2} h k x^{2} y c_{3}+\frac{1}{2} k^{2} y^{2} c_{3}+h x c_{4}+\frac{1}{2} h x^{2} c_{5}+k y c_{5},
\end{aligned}
$$

where $c_{i}, i=1, \ldots, 5$ are constants. Thus, the first integrals (conserved forms) for nonlinear fin equation (3.1) can be calculated by using expression (2.12) and by considering each group parameter $c_{i}$.

$$
\begin{aligned}
& I_{1}=h k y-\frac{1}{2} k^{2}\left(y^{\prime}\right)^{2}, \\
& I_{2}=\frac{1}{8}\left(-2 h^{2} x^{3}-4 h k x y+2 k\left(3 h x^{2}+2 k y\right) y^{\prime}-4 k^{2} x\left(y^{\prime}\right)^{2}\right), \\
& I_{3}=\frac{1}{8}\left(-h^{2} x^{4}-4 h k x^{2} y-4 k^{2} y^{2}+2 k\left(2 h x^{3}+4 k x y\right)\left(y^{\prime}\right)-4 k^{2} x^{2}\left(y^{\prime}\right)^{2}\right), \\
& I_{4}=-h x+k y^{\prime}, \quad I_{5}=-\frac{1}{2} h x^{2}-k y+k x y^{\prime} .
\end{aligned}
$$

Case 1.2: $H(y)=y$.

Based on the similar calculation in the first case, if we take $H(y)=y$, we obtain the infinitesimals $\xi$ and $\eta$ by solving equations $4 a^{\prime}(x)-k a^{\prime \prime \prime}(x)=0, b(x)-k b^{\prime \prime}(x)=0$

$$
\begin{aligned}
& \xi=k^{2}\left(\frac{1}{2} e^{\frac{-2 x}{\sqrt{k}}} \sqrt{k}\left(e^{\frac{4 x}{\sqrt{k}}} c_{1}-c_{2}\right)+c_{3}\right), \\
& \eta=\frac{1}{2} k^{2} y\left(2 e^{\frac{2 x}{\sqrt{k}}} c_{1}-e^{\frac{-2 x}{\sqrt{k}}}\left(e^{\frac{4 x}{\sqrt{k}}} c_{1}-c_{2}\right)\right)+k\left(e^{\frac{x}{\sqrt{k}}} c_{4}+e^{\frac{-x}{\sqrt{k}}} c_{5}\right),
\end{aligned}
$$


where $c_{i}, i=1, \ldots, 5$ are constants. The corresponding generators are

$$
\begin{aligned}
& X_{1}=\left(\frac{1}{2} e^{\frac{2 x}{\sqrt{k}}} k^{\frac{5}{2}}\right) \frac{\partial}{\partial x}+\left(\frac{1}{2} e^{\frac{2 x}{\sqrt{k}}} k^{2} y\right) \frac{\partial}{\partial y}, \\
& X_{2}=\left(-\frac{1}{2} e^{\frac{-2 x}{\sqrt{k}}} k^{\frac{5}{2}}\right) \frac{\partial}{\partial x}+\left(\frac{1}{2} e^{\frac{-2 x}{\sqrt{k}}} k^{2} y\right) \frac{\partial}{\partial y}, \\
& X_{3}=k^{2} \frac{\partial}{\partial x}, \quad X_{4}=\left(e^{\frac{x}{\sqrt{k}}} k\right) \frac{\partial}{\partial y}, \quad X_{5}=\left(e^{\frac{-x}{\sqrt{k}}} k\right) \frac{\partial}{\partial y},
\end{aligned}
$$

and the gauge function is

$$
B(x, y)=e^{\frac{-2 x}{\sqrt{k}}} \sqrt{k}\left(\frac{1}{2} e^{\frac{4 x}{\sqrt{k}}} k y^{2} c_{1}-\frac{1}{2} k y^{2} c_{2}+e^{\frac{3 x}{\sqrt{k}}} y c_{4}-e^{\frac{x}{\sqrt{k}}} c_{5} y\right)+c_{6},
$$

where $c_{6}$ is an arbitrary constant and the first integrals are found by using the expression (2.12)

$$
\begin{aligned}
& I_{1}=\frac{1}{4} e^{\frac{2 x}{\sqrt{k}}}\left(-k^{\frac{3}{2}} y^{2}+2 k^{2} y y^{\prime}-k^{\frac{5}{2}}\left(y^{\prime}\right)^{2}\right), \quad I_{2}=\frac{1}{4} e^{\frac{-2 x}{\sqrt{k}}}\left(k^{\frac{3}{2}} y^{2}+2 k^{2} y y^{\prime}+k^{\frac{5}{2}}\left(y^{\prime}\right)^{2}\right), \\
& I_{3}=\frac{1}{2}\left(k y^{2}-k^{2}\left(y^{\prime}\right)^{2}\right), \quad I_{4}=e^{\frac{x}{\sqrt{k}}}\left(k y^{\prime}-\sqrt{k} y\right), \\
& I_{5}=e^{\frac{-x}{\sqrt{k}}}\left(\sqrt{k} y+k y^{\prime}\right) .
\end{aligned}
$$

Case 1.3: $H(y)=y^{n}, n>1$.

In equation (3.19), if we take $H(y)=y^{n}$, then we obtain

$$
2 n y^{(-1+n)} b(x)+3 k y^{n} a^{\prime}(x)+k n y^{n} a^{\prime}(x)-2 k b^{\prime \prime}(x)-k^{2} y a^{\prime \prime \prime}(x)=0,
$$

which gives $a^{\prime}(x)=0$, and $b(x)=0$ gives $\xi$ and $\eta$

$$
\xi=k^{2} c_{1}, \quad \eta=0, \quad B(x, y)=0,
$$

where $c_{1}$ is a constant. Then the infinitesimal generator corresponding to (3.30) is

$$
X=k^{2} \frac{\partial}{\partial x}
$$

and the first integral is written similarly to the previous case

$$
I=-\frac{-2 k y^{1+n}+k^{2}(1+n)\left(y^{\prime}\right)^{2}}{2(1+n)} .
$$

Case 1.4: $H(y)=\operatorname{Exp}(y)$.

For this case, it is clear that the infinitesimal functions are

$$
\xi=k^{2} c_{1}, \quad \eta=0, \quad B(x, y)=0,
$$

where $c_{1}$ is a constant, and the generator is

$$
X=k^{2} \frac{\partial}{\partial x}
$$


and the first integral is

$$
I=e^{y} k-\frac{1}{2} k^{2}\left(y^{\prime}\right)^{2} .
$$

Case 1.5: $H(y)=\frac{1}{m y+n}$.

For this case, the infinitesimals are found as follows:

$$
\xi=k^{2} c_{1}, \quad \eta=0, \quad B(x, y)=0,
$$

where $c_{1}$ is an arbitrary constant, and the generator is

$$
X=k^{2} \frac{\partial}{\partial x}
$$

and the first integral is

$$
I=\frac{k \log (k(n+m y))}{m}-\frac{1}{2} k^{2}\left(y^{\prime}\right)^{2} .
$$

Case 1.6: Arbitrary function $H(y)$.

We find that

$$
\xi=k^{2} c_{1}, \quad \eta=0, \quad B(x, y)=0,
$$

where $c_{1}$ is a constant and the generator is

$$
X=k^{2} \frac{\partial}{\partial x}
$$

and the first integral is

$$
I=k \int H(y) d y-\frac{1}{2} k^{2}\left(y^{\prime}\right)^{2} .
$$

Case 2: $K(y)=k \operatorname{Exp}(\alpha y), k$ and $\beta$ are constants.

In equation (3.18), if we take $K(y)=k \operatorname{Exp}(\beta y)$, we obtain the following differential equation in terms of $H(y)$ function

$$
\left(2 \alpha b(x)+a^{\prime}(x) e^{y \alpha} k\right) H^{\prime}(y)+3 e^{y \alpha} k \alpha a^{\prime}(x) H(y)-e^{y \alpha} k\left(2 \alpha b^{\prime \prime}(x)+e^{y \alpha} k a^{\prime \prime \prime}(x)\right)=0,
$$

and consider the following cases as the solutions of (3.42), and we get the mathematical relations between functions $a(x)$ and $b(x)$.

Case 2.1: $H(y)=h($ constant $)$.

For this case, differential equation (3.42) yields

$$
e^{y \alpha} k\left(3 h \alpha a^{\prime}(x)+2 \alpha b^{\prime \prime}(x)+e^{y \alpha} k a^{\prime \prime \prime}(x)\right)=0 .
$$


In (3.43) $k \neq 0$, then the term in the parenthesis must be zero, which gives $a^{\prime \prime \prime}(x)=0$ and $3 h a^{\prime}(x)+2 b^{\prime \prime}(x)=0$, then the infinitesimal functions are found as follows:

$$
\begin{aligned}
& \xi=e^{2 y \alpha} k^{2}\left(c_{1}+x c_{2}+x^{2} c_{3}\right), \\
& \eta=\frac{e^{2 y \alpha} k^{2}\left(c_{2}+2 x c_{3}\right)}{2 \alpha}+e^{y \alpha} k\left(\frac{3}{4} h x^{2} c_{2}+\frac{1}{2} h x^{3} c_{3}+c_{4}+x c_{5}\right),
\end{aligned}
$$

where $c_{i}, i=1, \ldots, 5$ are constants, and we have following five infinitesimal generators:

$$
\begin{aligned}
& X_{1}=\left(e^{2 y \alpha} k^{2}\right) \frac{\partial}{\partial x}, \quad X_{2}=\left(e^{2 y \alpha} k^{2} x\right) \frac{\partial}{\partial x}+\left(\frac{3}{4} e^{y \alpha} h k x^{2}+\frac{e^{2 y \alpha} k^{2}}{2 \alpha}\right) \frac{\partial}{\partial y}, \\
& X_{3}=\left(e^{2 y \alpha} k^{2} x^{2}\right) \frac{\partial}{\partial x}+\left(\frac{1}{2} e^{y \alpha} h k x^{3}+\frac{e^{2 y \alpha} k^{2} x}{\alpha}\right), \\
& X_{4}=e^{y \alpha} k \frac{\partial}{\partial y}, \quad X_{5}=e^{y \alpha} k x \frac{\partial}{\partial y},
\end{aligned}
$$

and we have the gauge function

$$
\begin{aligned}
B(x, y)= & \frac{1}{8 \alpha^{2}} 4\left(e^{2 y \alpha} k^{2} c_{3}-4 e^{y \alpha} k \alpha\left(h\left(4 c_{1}+x\left(c_{2}+x c_{3}\right)\right)-2 c_{5}\right)\right. \\
& \left.+h x \alpha^{2}\left(h x^{2}\left(2 c_{2}+x c_{3}\right)+8 c_{4}+4 x c_{5}\right)\right),
\end{aligned}
$$

and the corresponding first integrals

$$
\begin{aligned}
I_{1}= & \frac{e^{\alpha y} h k}{\alpha}-\frac{1}{2} e^{2 \alpha y} k^{2} \alpha^{2}\left(y^{\prime}\right)^{2}, \\
I_{2}= & -\frac{2 e^{y} h k x \alpha+h^{2} x^{3} \alpha^{2}-e^{\alpha y} k \alpha\left(2 e^{\alpha y} k+3 h x^{2} \alpha\right) y^{\prime}+2 e^{2 \alpha y} k^{2} x \alpha^{2}\left(y^{\prime}\right)^{2}}{4 \alpha^{2}}, \\
I_{3}= & -\left(4 e^{2 \alpha y} k^{2}+4 e^{\alpha y} h k x^{2} \alpha+h^{2} x^{4} \alpha^{2}-2 e^{\alpha y} k \alpha\left(4 e^{\alpha y} k x+2 h x^{3} \alpha\right)\left(y^{\prime}\right)\right. \\
& \left.+4 e^{2 \alpha y} k^{2} x^{2} \alpha^{2}\left(y^{\prime}\right)^{2}\right) /\left(8 \alpha^{2}\right), \\
I_{4}= & e^{\alpha y} k \alpha^{2} y^{\prime}-h x, \quad I_{5}=e^{\alpha y} k x y^{\prime}-\frac{1}{2} h x^{2}-\frac{e^{\alpha y} k}{\alpha} .
\end{aligned}
$$

Case 2.2: Arbitrary $H(y)$.

For an arbitrary $H(y)$ function, we obtain infinitesimal functions in the form

$$
\xi=e^{2 y \alpha} k^{2} c_{1}, \quad \eta=0,
$$

where $c_{1}$ is a constant, and the infinitesimal generator is

$$
X=e^{2 \alpha y} k^{2} \frac{\partial}{\partial x}
$$

and the gauge function is

$$
B(x, y)=2 k \alpha c_{1} \int e^{2 \alpha y}\left(\int e^{-\alpha y} H(y) d y\right) d y,
$$


and the first integral is calculated as follows:

$$
\begin{aligned}
I= & k\left(e^{2 \alpha y} \int e^{-\alpha y} H(y) d y\right. \\
& \left.-2 \alpha \int e^{2 \alpha y}\left(\int e^{-\alpha y} H(y) d y\right) d y-\frac{1}{2} e^{2 \alpha y} k\left(y^{\prime}\right)^{2}\right) .
\end{aligned}
$$

Case 2.3: $H(y)=\frac{h}{(\beta y+\gamma)^{2}}, \beta$ and $\gamma$ are arbitrary constants.

For this case, the infinitesimals $\xi$ and $\eta$ are

$$
\xi=e^{2 y \alpha} k^{2} c_{1}, \quad \eta=0,
$$

where $c_{1}$ is a constant and the infinitesimal generator is

$$
X=e^{2 \alpha y} k^{2} \frac{\partial}{\partial x},
$$

and the gauge function is

$$
\begin{aligned}
B(x, y)= & -\frac{e^{-\frac{\alpha \gamma}{\beta}} h k \alpha c_{1}}{\beta^{2}}\left(e^{\frac{2 \alpha(\gamma \beta+\gamma)}{\beta}} \operatorname{ExpIntegralEi}\left(-\frac{\alpha(y \beta+\gamma)}{\beta}\right)\right. \\
& \left.+ \text { ExpIntegralEi }\left(\frac{\alpha(y \beta+\gamma)}{\beta}\right)\right),
\end{aligned}
$$

where ExpIntegralEi is a special function on the complex plane. For real nonzero values of $x$, the exponential integral $\operatorname{Ei}(x)$ is defined as

$$
\operatorname{Ei}(x)=\int_{-\infty}^{x}\left(\frac{e^{t}}{t}\right) d t
$$

and the first integral is

$$
\begin{aligned}
I= & \frac{1}{2 \beta^{2}(\gamma+\beta y)} e^{-\frac{\alpha \gamma}{\beta}}\left(-2\left(e^{\frac{\alpha \gamma}{\beta}+\alpha y} h k \beta-h k \alpha \operatorname{ExpIntegralEi}\left(\alpha\left(\frac{\gamma}{\beta}+y\right)\right)(\gamma+\beta y)\right)\right. \\
& \left.-e^{-\frac{\alpha \gamma}{\beta}+2 \alpha y} k^{2} \beta^{2}(\gamma+\beta y)\left(y^{\prime}\right)^{2}\right) .
\end{aligned}
$$

Case 3: $K(y)=k y^{\beta}, \beta \neq-1$.

If we take $H(y)=h$ is constant, then we obtain the following equation:

$$
-3 h(1+\beta) a^{\prime}(x)+2(1+\beta) b^{\prime \prime}(x)+k y^{1+\beta} a^{\prime \prime \prime}(x)=0,
$$

and we find the infinitesimal functions from the solutions of $a^{\prime \prime \prime}(x)=0$ and $-3 h(1+\beta) a^{\prime}(x)+$ $2(1+\beta) b^{\prime \prime}(x)=0$

$$
\begin{aligned}
& \xi=k^{2} y^{2 \beta}\left(c_{1}+x c_{2}+x^{2} c_{3}\right), \\
& \eta=\frac{k^{2} y^{1+2 \beta}\left(c_{2}+2 x c_{3}\right)}{2(1+\beta)}+k y^{\beta}\left(\frac{3}{4} h x^{2} c_{2}+\frac{1}{2} x^{3} c_{3}+c_{4}+x c_{5}\right),
\end{aligned}
$$


where $c_{i}, i=1, \ldots, 5$ are constants. In this case, we have the following five infinitesimal generators:

$$
\begin{aligned}
& X_{1}=k^{2} y^{2} \beta \frac{\partial}{\partial x}, \quad X_{2}=k^{2} x y^{2 \beta} \frac{\partial}{\partial x}+\left(\frac{3}{4} h k x^{2} y^{\beta}+\frac{k^{2} y^{1+2 \beta}}{2(1+2 \beta)}\right) \frac{\partial}{\partial y}, \\
& X_{3}=k^{2} x^{2} y^{2 \beta} \frac{\partial}{\partial x}+\left(\frac{1}{2} k x^{3} y^{\beta}+\frac{k^{2} x y^{1+2 \beta}}{(1+2 \beta)}\right) \frac{\partial}{\partial y}, \\
& X_{4}=k y^{\beta} \frac{\partial}{\partial y}, \quad X_{5}=x k y^{\beta} \frac{\partial}{\partial y},
\end{aligned}
$$

and the gauge function is

$$
\begin{aligned}
B(x, y)= & \frac{1}{8}\left(h x\left(h x^{2}\left(2 c_{2}+x c_{3}\right)+8 c_{4}+4 x c_{5}\right)+\frac{4 k y^{1+\beta}}{(\beta-1)(\beta+1)^{2}}\left(-h(1+\beta)\left(3 x\left(c_{2}+x c_{3}\right)\right.\right.\right. \\
& \left.\left.\left.+\beta\left(4 c_{1}+x\left(c_{2}+x c_{3}\right)\right)\right)+(\beta-1)\left(k y^{\beta} c_{3}+2(\beta+1) c_{5}\right)\right)\right) .
\end{aligned}
$$

Using equation (3.59), we obtain three first integrals

$$
\begin{aligned}
& I_{1}=-\frac{k y^{\beta}\left(-2 h y+k(1+\beta) y^{\beta} y^{\prime 2}\right)}{2(1+\beta)}, \\
& I_{2}=-\frac{1}{4(1+\beta)}\left(\left(h x-k y^{\beta} y^{\prime}\right)\left(h x^{2}(1+\beta)+2 k y^{1+\beta}-2 k x(1+\beta) y^{\beta} y^{\prime}\right)\right), \\
& I_{3}=-\frac{1}{8(1+\beta)^{2}}\left(h x^{2}(1+\beta)+2 k y^{1+\beta}-2 k x(1+\beta) y^{\beta} y^{\prime}\right)^{2}, \\
& I_{4}=-h x+k y^{\beta} y^{\prime}, \quad I_{5}=\frac{h x^{2}}{2}-\frac{k y^{(1+\beta)}}{1+\beta}+k x y^{\beta} y^{\prime} .
\end{aligned}
$$

Case 3.1: $K(y)=k y^{\beta}, \beta=-1$.

For this case, equation (3.18) is equal to

$$
3 h a^{\prime}(x)-2 b^{\prime \prime}(x)-k a^{\prime \prime \prime}(x) \ln y=0,
$$

and by using (3.61), the infinitesimals functions become

$$
\begin{aligned}
\xi & =\frac{k^{2}}{y^{2}}\left(c_{1}+x c_{2}+x^{2} c_{3}\right), \\
\eta & =\frac{1}{y}\left(k\left(\frac{3}{4} h x^{2} c_{2}+\frac{1}{2} h x^{3} c_{3}+c_{4}+x c_{5}\right)+k^{2}\left(c_{2}+2 x c_{3}\right) \ln y\right),
\end{aligned}
$$

where $c_{i}, i=1, \ldots, 5$ are constants and the infinitesimal generators are

$$
\begin{aligned}
& X_{1}=\frac{k^{2}}{y^{2}} \frac{\partial}{\partial x}, \quad X_{2}=\frac{k^{2} x}{y^{2}} \frac{\partial}{\partial x}+\frac{k\left(3 h x^{2}+2 k \ln y\right)}{4 y} \frac{\partial}{\partial y}, \\
& X_{3}=\frac{k^{2} x^{2}}{y^{2}} \frac{\partial}{\partial x}+\frac{k\left(2 h x^{3}+4 h k x \ln y\right)}{4 y} \frac{\partial}{\partial y}, \\
& X_{4}=\frac{k}{y} \frac{\partial}{\partial y}, \quad X_{5}=\frac{k x}{y} \frac{\partial}{\partial y},
\end{aligned}
$$


and the gauge function is

$$
\begin{aligned}
B(x, y)= & \frac{1}{8}\left(h x\left(4 k\left(c_{2}+x c_{3}\right)+h x^{2}\left(2 c_{2}+x c_{3}\right)+8 c_{4}+4 x c_{5}\right)\right. \\
& \left.+4 k\left(h\left(-2 c_{1}+x\left(c_{2}+x c_{3}\right)+2 c_{5}\right) \ln y+4 k^{2} c_{3} \ln y^{2}\right)\right),
\end{aligned}
$$

and we have four first integrals

$$
\begin{aligned}
& I_{1}=\frac{k}{2}\left(h+2 h \ln y-\frac{k y^{\prime 2}}{y^{2}}\right), \\
& I_{2}=\frac{k y^{\prime}-h x y}{4 y^{2}}\left(\left(h x^{2}+2 k \ln y\right) y-2 k x y^{\prime}\right), \\
& I_{3}=-\frac{1}{8 y^{2}}\left(\left(h x^{2}+2 k \ln y\right) y-2 k x y^{\prime}\right)^{2}, \\
& I_{4}=\frac{k y^{\prime}}{y}-h x, \quad I_{5}=k x \frac{y^{\prime}}{y}-k \ln y-\frac{h x^{2}}{2} .
\end{aligned}
$$

\section{Invariant solutions}

Some group invariant solutions of nonlinear fin equation (3.1) can be constructed from the Noether symmetries and the first integrals. In this section, we consider some different special cases to present invariant solutions of (3.1).

Case 1. For the case $K(y)=k($ constant $)$ and $H(y)=h($ constant $)$, the first conservation law is

$$
I=h k y-\frac{1}{2} k^{2}\left(y^{\prime}\right)^{2}
$$

Then the expression $D_{x} I=0$ gives the following invariant solution of fin equation (3.1):

$$
y(x)=\frac{4 c+2 h^{2} x^{2}-2 \sqrt{2} h^{2} k x c_{1}+h^{2} k^{2} c_{1}^{2}}{4 h k},
$$

where $c, c_{1}$ are constants.

Case 2. As another case, if we consider $K(y)=k$ (constant) and $H(y)=y$, then the first integral becomes

$$
I=\frac{1}{4} e^{\frac{-2 x}{\sqrt{k}}}\left(k^{\frac{3}{2}} y^{2}+2 k^{2} y y^{\prime}+k^{\frac{5}{2}}\left(y^{\prime}\right)^{2}\right),
$$

and $D_{x}=0$ yields the following solution:

$$
y(x)=-\sqrt{c} \frac{e^{\frac{x}{\sqrt{k}}}}{k^{\frac{3}{4}}}+e^{-\frac{x}{\sqrt{k}}} c_{2},
$$

where $c, c_{2}$ are constants. This solution (4.4) is the group invariant solution that satisfies the original fin equation (3.1). 
Table 1 Noether symmetry classification table of fin equation

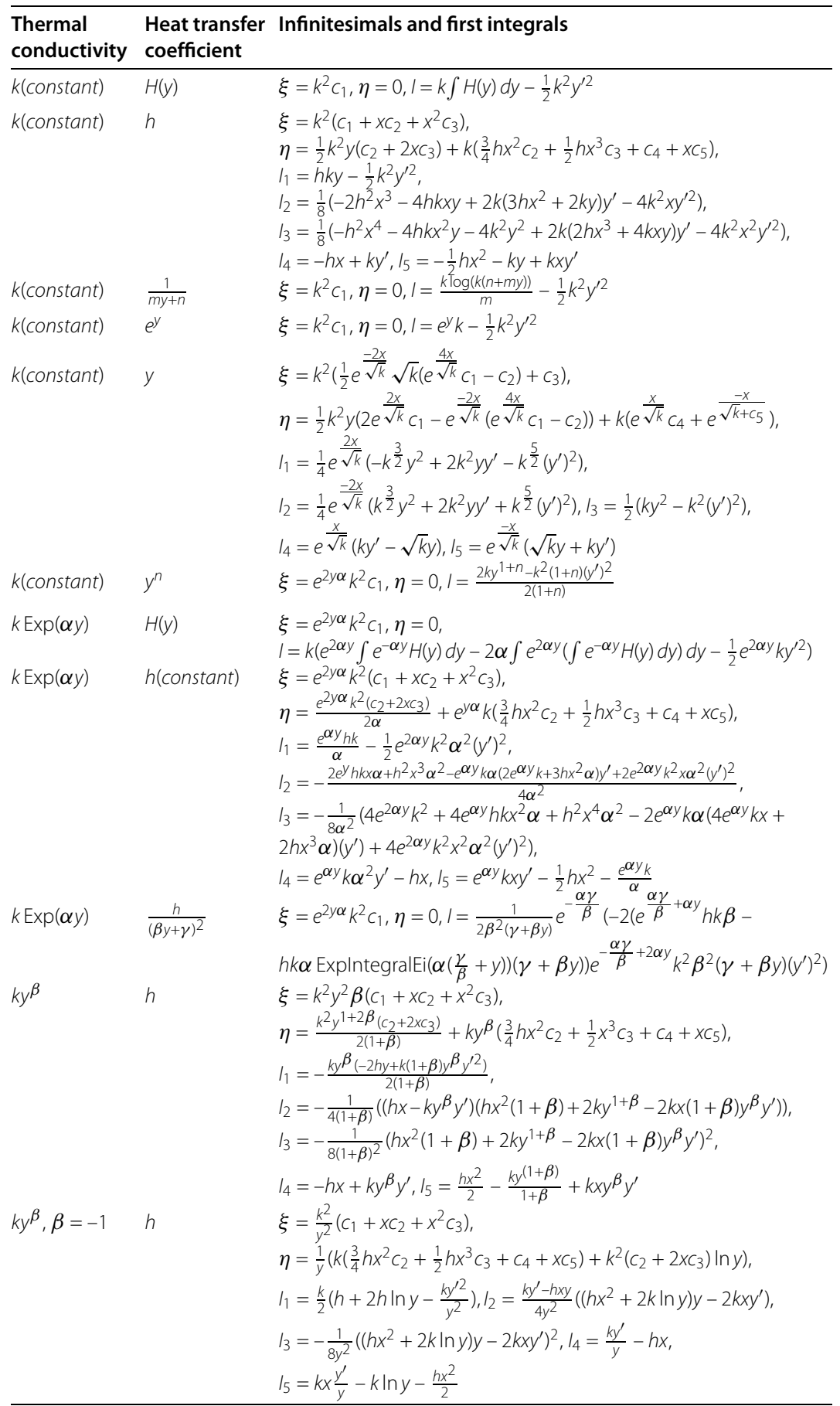

Case 3. As the third case, we consider $K(y)=k($ constant $)$ and $H(y)=y$ and find the conserved form as follows:

$$
I=\frac{1}{2} e^{\frac{-2 x}{\sqrt{k}}}\left(e^{\frac{2 x}{\sqrt{k}}} k y^{2}-e^{\frac{2 x}{\sqrt{k}}} k^{2}\left(y^{\prime}\right)^{2}\right)
$$


and (4.5) gives the following invariant solution:

$$
y(x)=\frac{e^{-\frac{x+k c_{3}}{\sqrt{k}}}\left(e^{2 \sqrt{k} c_{3}}+8 c e^{\frac{2 x}{\sqrt{k}}}\right)}{4 k} .
$$

Case 4. The choice of $K(y)=k \operatorname{Exp}(\alpha y)$ and $H(y)=h$ (constant) yields the conservation law

$$
I=\frac{e^{\alpha y} h k}{\alpha}+\frac{1}{2} e^{2 \alpha y} k^{2} \alpha^{2}\left(y^{\prime}\right)^{2},
$$

and by integration of (4.8), we find the group invariant solution in the following form:

$$
y(x)=\frac{1}{\alpha} \ln \left(\frac{c \alpha}{h k}+\frac{1}{2 k} h x^{2} \alpha+\frac{h x \alpha^{\frac{3}{2}}}{\sqrt{2}} c_{4}+\frac{1}{4} h k \alpha^{2} c_{4}^{2}\right)
$$

where $c_{4}$ is constant, which satisfies fin equation (3.1).

\section{Concluding remarks}

In this study we analyze the Noether symmetry group classification of a nonlinear fin equation, which is a second-order nonlinear ordinary differential equation. Here, we consider thermal conductivity and heat transfer coefficient as variable functions of temperature, and the nonlinear fin equation is considered in a one-dimensional model describing heat transfer in rectangular fins. From the mathematical point of view, it can be said that this problem is highly nonlinear. Here, we consider applying a partial Lagrangian approach for the classification to this problem. For different heat transfer coefficient and thermal conductivity functions, we obtain Noether point symmetry algebras. Finally, we find the corresponding new first integrals for each case, the results are presented in a Table 1, and for each case, some invariant solutions are obtained from the first integrals (conserved forms). This study can be considered as one of the first studies on the Noether symmetry classification of differential equations in the literature. In addition, it is important to mention that the $\lambda$-symmetry method is another new approach to finding first integrals for differential equations. As a further study, we will deal with the $\lambda$-symmetry classification of the same problem. This study is still in progress.

Competing interests

The authors declare that they have no competing interests.

Authors' contributions

All authors contributed equally to the manuscript and jointly worked on the results. All authors typed, read and approved the final manuscript. The authors are very grateful to both reviewers for carefully reading this paper and for their comments.

Author details

${ }^{1}$ Department of Mathematics, Faculty of Science and Letters, İstanbul Technical University, Maslak, İstanbul, 34469, Turkey.

${ }^{2}$ Division of Mechanics, Faculty of Civil Engineering, i̇stanbul Technical University, Maslak, İstanbul, 34469, Turkey.

Acknowledgements

Dedicated to Professor Hari M Srivastava.

Received: 12 December 2012 Accepted: 12 March 2013 Published: 3 April 2013 


\section{References}

1. Noether, E: Invariante Variationsprobleme. Nachr. Ges. Wiss. Gött., Math.-Phys. Kl. 2, 235-257 (1918). (English translation in Transport Theory and Statistical Physics 1(3), 186-207 (1971))

2. Bluman, GW, Kumei, S: Symmetries and Differential Equations. Springer, Berlin (1989)

3. Ovsiannikov, LV: Group Analysis of Differential Equations. Nauka, Moscow (1978)

4. Ibragimov, NH (ed.): CRC Handbook of Lie Group Analysis of Differential Equations, vol. I (1994)

5. Ibragimov, $\mathrm{NH}$ (ed.): CRC Handbook of Lie Group Analysis of Differential Equations, vol. II (1995)

6. Ibragimov, NH (ed.): CRC Handbook of Lie Group Analysis of Differential Equations, vol. III (1996)

7. Olver, PJ: Applications of Lie Groups to Differential Equations. Springer, Berlin (1986)

8. Stephani, H: Differential Equations: Their Solutions Using Symmetries. Cambridge University Press, Cambridge (1989)

9. Özer, T: Symmetry group classification for one-dimensional elastodynamics problems in nonlocal elasticity. Mech. Res. Commun. 30(6), 539-546 (2003)

10. Özer, T: On symmetry group properties and general similarity forms of the Benney equations in the Lagrangian variables. J. Comput. Appl. Math. 169(2), 297-313 (2004)

11. Özer, T: Symmetry group classification for two-dimensional elastodynamics problems in nonlocal elasticity. Int. J. Eng Sci. 41(18), 2193-2211 (2003)

12. Kara, AH, Mahomed, FM, Naeem, I, Wafo Soh, C: Partial Noether operators and first integrals via partial Lagrangians. Math. Methods Appl. Sci. 30, 2079-2089 (2007)

13. Ibragimov, NH, Kara, AH, Mahomed, FM: Lie-Backlund and Noether symmetries with applications. Nonlinear Dyn. 15, 115-136 (1998)

14. Levy-Leblond, JM: Conservation laws for Gauge invariant Lagrangians in classical mechanics. Am. J. Phys. 11, 249-258 (1978)

15. Kara, AH, Mahomed, FM, Naeem, I, Leach, PGL: Lie and Noether counting theorems for one-dimensional systems. J. Math. Anal. Appl. 78, 116-129 (1993)

16. Kim, S, Moon, JH, Huang, CH: An approximate solution of the nonlinear fin problem with temperature-dependent thermal conductivity and heat transfer coefficient. J. Phys. D, Appl. Phys. 40, 2979-2987 (2007)

17. Moitsheki, RJ, Harley, C: Transient heat transfer in longitudinal fins of various profiles with temperature-dependent thermal conductivity and heat transfer coefficient. Pramana 77(3), 519-532 (2011)

18. Moitsheki, RJ, Mhlongo, MD: Classical Lie point symmetry analysis of a steady nonlinear one-dimensional fin problem. J. Appl. Math. 2012, Article ID 671548 (2012)

doi:10.1186/1029-242X-2013-147

Cite this article as: Orhan et al.: On symmetry group classification of a fin equation. Journal of Inequalities and Applications 2013 2013:147.

\section{Submit your manuscript to a SpringerOpen ${ }^{\odot}$ journal and benefit from:}

- Convenient online submission

- Rigorous peer review

Immediate publication on acceptance

Open access: articles freely available online

- High visibility within the field

- Retaining the copyright to your article 